\title{
Coupling-mass mapping of di-jet peak searches
}

\author{
Bogdan A. Dobrescu* and Felix Yut \\ Theoretical Physics Department, Fermilab, Batavia, IL 60510, USA
}

(Dated: June 11, 2013; Revised September 10, 2014)

\begin{abstract}
We study hypothetical gauge bosons that may produce dijet resonances at the LHC. Simple renormalizable models include leptophobic $Z^{\prime}$ bosons or colorons that have flavorindependent couplings and decay into a color-singlet or -octet quark-antiquark pair, respectively. We present the experimental results on dijet resonances at hadron colliders as limits in the coupling-versus-mass plane of a gauge boson associated with baryon number. This theoretical framework facilitates a direct comparison of dijet resonance searches performed at different center-of-mass energies or at different colliders.
\end{abstract}

\section{INTRODUCTION}

If a new particle is produced in the $s$-channel at hadron colliders, then it can decay into a pair of hadronic jets ("dijet"). The invariant mass distribution of the dijet exhibits a peak at (or slightly below) the mass of the new particle [1, 2]. In many models, particles produced in the $s$-channel can decay into leptons or other final states with low backgrounds. If the branching fractions of those final states are small enough, however, the dijet resonance searches may provide the simplest way of discovering the new particles.

Searches for narrow dijet resonances at hadron colliders have been performed over the last three decades by the UA2 [3, 4] and UA1 [5] experiments at the SPS collider, the CDF [ $6-10]$ and D0 [11] experiments at the Tevatron, and the

\footnotetext{
*bdob@fnal.gov

† felixyu@fnal.gov
}

ATLAS [12 19] and CMS [20 25] experiments at the LHC. The results are traditionally presented as limits on an effective rate (defined as cross section times branching fraction times acceptance) to produce a resonance as a function of its mass. While this procedure has the advantage of being rather model-independent, it complicates the comparison of experimental results with theoretical models.

The acceptance, in particular, requires the computation of the probability for the two jets to be observed in a certain geometric region of the detector. This can be done analytically given the differential cross section of the signal and the kinematic cuts, as long as effects arising from showering, from an assumed Gaussian signal (in the case of ATLAS [16]), or from a mismatch between partons and analysis-level wide jets are negligible. Otherwise, it is necessary to perform a simulation based on the jet selection criteria used by the experimental analyses. 
The effective rate procedure also precludes a comparison of the limits set in $p \bar{p}$ collisions (at the SPS and the Tevatron) with those from $p p$ collisions (at the LHC). Even for a particular collider, it is hard to compare the limits set during runs of different energies, because the cross section grows with the center-of-mass energy $(\sqrt{s})$ for a fixed resonance mass. A naive hope is that limits set at the larger $\sqrt{s}$ and with larger integrated luminosity would supersede previous limits. The situation is not so straightforward because the backgrounds also increase so that the trigger thresholds for a jet-only final state need to be increased. As a result, the sensitivity to lighter resonances can be better in the runs using lower luminosity or lower energy. For example, the ATLAS dijet limits from $\sqrt{s}=7 \mathrm{TeV}$ start at a resonance mass that has increased with luminosity from $0.3 \mathrm{TeV}$ [12] to $0.6 \mathrm{TeV}$ [13] to 0.9 $\mathrm{TeV}$ [16] to $1 \mathrm{TeV}$ [17], and those from $\sqrt{s}=8$ $\mathrm{TeV}$ start at a mass $1.5 \mathrm{TeV}$ [18, 19].

In this article we explore a unified presentation of the dijet limits in a coupling-versus-mass plot. The mass and coupling refer to a certain hypothetical particle, of a given spin and $S U(3)_{c} \times S U(2)_{W} \times U(1)_{Y}$ charges, which is produced in the $s$-channel at hadron colliders and decays into a pair of jets. This is by no means a substitute for the effective rate plots, as it is more model-dependent. The coupling-versusmass plot, however, has the advantage of allowing simple comparisons of searches performed at dif- ferent luminosities, experiments, $\sqrt{s}$ or colliders. Furthermore, it provides a measure of how stringent the limits are given some natural ranges for the physical parameters.

Specifically, we consider an electricallyneutral spin-1 particle that couples in a flavoruniversal way to the SM quark-antiquark pairs and is leptophobic, i.e., its tree-level couplings to SM leptons vanish. This is well motivated by the following arguments. In many theories beyond the SM, there are particles that can be produced from a quark-antiquark initial state and lead to a dijet resonance with large rates. By contrast, both the gluon-gluon (as in the case of the Higgs boson) and quark-gluon initial states require a loop to produce an $s$-channel resonance, so that the signal is typically too small (at least in perturbative theories) to compete with the dijet background. The quark-quark initial state could lead to an $s$-channel resonance if there is a di-quark scalar, but in that case flavorchanging processes typically impose strong constraints on its mass and couplings (these are relaxed in the case of the color-sextet, hypercharge4/3 di-quark [26]).

Electroweak symmetry suppresses the coupling of spin-0 particles to first generation $q \bar{q}$ pairs (an exception is the color-octet weakdoublet scalar [27], but in that case there are strong flavor constraints). Leptophobic spin-2 particles, although possible, require much more complicated UV completions. 
Including a spin-1 particle coupled to first generation quarks is more straightforward. Large flavor effects are avoided if its quark couplings are generation-independent. Moreover, the spin-1 particle should be associated with a spontaneously-broken gauge symmetry (unless the particle is a bound state whose compositeness scale is near its mass), and the cancellation of various gauge anomalies is more easily achieved for equal couplings to up- and downtype quarks. Although some of the above arguments can be evaded (for example, with a more complicated fermion sector that is anomaly-free), a flavor-universal gauge boson appears to be the simplest origin of a dijet peak. In order to couple to the SM quarks, the heavy gauge boson must be either a singlet or an octet under the $S U(3)_{c}$ color group.

In the case of the color singlet (a $Z^{\prime}$ boson), the dijet channel can be the discovery mode only if the $Z^{\prime}$ is nearly leptophobic (for an early model, see [28]) and its decays into Higgs states [29] or vectorlike leptons [30] are suppressed. We consider $Z^{\prime}$ bosons whose tree-level leptonic and Higgs couplings vanish, implying that the gauge charges are proportional to the baryon number. The corresponding $U(1)_{B}$ symmetry is anomalous in the SM, but we will show that it is anomaly-free in the presence of a few vector-like quarks (the simplest charge assignment has been discussed in [31]).

A color-octet gauge boson, referred to as the coloron [32], is associated with a $S U(3)_{1} \times S U(3)_{2}$ extension of QCD, and is automatically leptophobic. The coloron, in the case of flavor-universal couplings [33], can arise from a simple renormalizable extension of the SM [34]. Although its low-energy effects are usually negligible (in contrast to the case of flavor-dependent couplings [35]), the coloron can modify Higgs production via gluon-fusion [36].

In Section [II we present some simple renormalizable models that include a $Z^{\prime}$ boson coupled to baryon number $\left(Z_{B}^{\prime}\right)$ or a coloron $\left(G^{\prime}\right)$. In Section III we use the existing experimental limits on the effective rate to derive the limits in the coupling-mass plane for $Z_{B}^{\prime}$, and also for $G^{\prime}$. Section IV includes our conclusions.

\section{MODELS OF DIJET RESONANCES}

In this section we present some renormalizable models of spin-1 particles that are either color singlets $\left(Z^{\prime}\right)$ or octets (coloron) and couple to quark-antiquark pairs.

\section{A. $Z^{\prime}$ coupled to baryon number}

Each coupling of a $Z^{\prime}$ boson to a quark or a lepton is in principle a free parameter. In practice, though, there are various theoretical and phenomenological constraints on these couplings. Massive spin-1 particles, such as $Z^{\prime}$ bosons, must be either bound states or else be associated with a spontaneously-broken gauge symmetry, the sim- 
plest case being a new $U(1)$ group. At hadron colliders, in order to discover a dijet resonance before a dilepton resonance, some of the $Z^{\prime}$ couplings to quarks must be more than an order of magnitude larger than all $Z^{\prime}$ couplings to leptons.

In the limit where the tree-level couplings to leptons vanish, the case of a leptophobic $Z^{\prime}$, there are severe constraints from the requirement of anomaly cancellations. These form a set of linear, quadratic and cubic equations in the $U(1)$ charges, which must have as a solution a set of commensurate numbers (i.e., a set of integers upon a rescaling of the gauge coupling). Despite the intricacies of cubic equations for integers, it can be proven that there is always a solution in the presence of a certain set of fermions which are vectorlike with respect to the SM gauge group and chiral under $U(1)$ [37]. To have a viable model, however, the number of new fermions cannot be too large, and their properties must avoid various phenomenological constraints.

Let us construct some viable models where the $U(1)_{B}$ symmetry associated with baryon number is gauged, i.e., all SM quarks have $U(1)_{B}$ charge $1 / 3$, while all SM leptons and bosons have charge 0 . This choice is convenient because the SM mechanism for generating quark masses is not affected by the additional gauge symmetry, and furthermore the $Z^{\prime}$ couplings to quarks are flavor blind.

We construct a class of explicit models of this type that include $n$ sets of vectorlike quarks (color-triplets) transforming under $S U(2)_{W}$ as doublets, $Q^{k}$, or singlets, $U^{k}, D^{k}$; here $k=$ $1, \ldots, n$ labels their flavor. Although these new fermions do not introduce anomalies involving only SM gauge groups, the $U(1)_{B}$ charges of the vectorlike quarks are restricted by anomaly cancellation. The $\left[S U(2)_{W}\right]^{2} U(1)_{B},\left[S U(3)_{c}\right]^{2} U(1)_{B}$ and $\left[U(1)_{Y}\right]^{2} U(1)_{B}$ anomalies cancel only if

$$
z_{U_{L}}-z_{U_{R}}=z_{D_{L}}-z_{D_{R}}=-z_{Q_{L}}+z_{Q_{R}}=\frac{1}{n},
$$

where $z_{Q_{R}}$ is the $U(1)_{B}$ charge of $Q_{R}$, etc. The $U(1)_{Y}\left[U(1)_{B}\right]^{2}$ anomaly then cancels only if

$$
z_{Q_{L}}=2 z_{U_{R}}-z_{D_{R}}
$$

It follows that there is no $U(1)_{B}$ gaugegravitational anomaly, and finally, the $\left[U(1)_{B}\right]^{3}$ anomaly cancels only if

$$
\left(z_{U_{R}}-z_{D_{R}}\right)\left(7 z_{U_{R}}-z_{D_{R}}+3 / n\right)=0
$$

We will refer to the $z_{D_{R}}=z_{U_{R}}$ solution as the $D=U$ model, and to the $z_{D_{R}}=7 z_{U_{R}}+3 / n$ solution as the $D=7 U+3$ model. Both these models are in fact families of $U(1)_{B}$ charges for the vectorlike quarks described by a rational parameter $\left(z_{U_{R}} \equiv z\right)$ and an integer $n$ (the number of vectorlike flavors).

There is need for at least one scalar field, $\phi$, to carry $U(1)_{B}$ charge and to have a VEV. The 'vectorlike' quarks are chiral with respect to $U(1)_{B}$, so that they can acquire mass only by coupling to the VEVs that break $U(1)_{B}$. In renormalizable models with only one $\phi$ scalar, Eq. (10) then requires that the charge of $\phi$ is $+1 / n$ (charge $-1 / n$ 


\begin{tabular}{|c|c|c|c|c|}
\hline \multirow[t]{2}{*}{ field } & \multirow{2}{*}{\multicolumn{2}{|c|}{$S U(2)_{W} U(1)_{Y}$}} & \multicolumn{2}{|c|}{$U(1)_{B}$} \\
\hline & & & $D=U$ model & $D=7 U+3$ model \\
\hline$u_{R}^{j}$ & 1 & $+2 / 3$ & \multicolumn{2}{|c|}{$+1 / 3$} \\
\hline$d_{R}^{j}$ & 1 & $-1 / 3$ & \multicolumn{2}{|c|}{$+1 / 3$} \\
\hline$q_{L}^{j}$ & 2 & $+1 / 6$ & \multicolumn{2}{|c|}{$+1 / 3$} \\
\hline $\begin{array}{l}U_{L}^{k} \\
U_{R}^{k}\end{array}$ & 1 & $+2 / 3$ & \multicolumn{2}{|c|}{$\begin{array}{c}z+1 / n \\
z\end{array}$} \\
\hline $\begin{array}{l}D_{L}^{k} \\
D_{R}^{k}\end{array}$ & 1 & $-1 / 3$ & $\begin{array}{c}z+1 / n \\
z\end{array}$ & $\begin{array}{l}7 z+4 / n \\
7 z+3 / n\end{array}$ \\
\hline $\begin{array}{l}Q_{L}^{k} \\
Q_{R}^{k}\end{array}$ & 2 & $+1 / 6$ & $\begin{array}{c}z \\
z+1 / n\end{array}$ & $\begin{array}{l}-5 z-3 / n \\
-5 z-2 / n\end{array}$ \\
\hline$\phi$ & 1 & 0 & \multicolumn{2}{|c|}{$+1 / n$} \\
\hline
\end{tabular}

TABLE I. Fields carrying $U(1)_{B}$ charge. With the exception of $\phi$ (a color-singlet scalar), all fields shown here are color-triplet fermions. The charge assignments labelled by $D=U$ and $D=7 U+3$ correspond to the two solutions of the $\left[U(1)_{B}\right]^{3}$ anomaly cancellation condition. The SM quarks have a generation index, $j=1,2,3$, and the vectorlike quarks have a flavor index $k=1, \ldots, n$.

is the same modulo the interchange of $\phi$ and $\phi^{\dagger}$ ) so that operators of the type $\bar{Q}_{L} Q_{R} \phi^{\dagger}$ are gauge invariant. The fields charged under $U(1)_{B}$ are listed in Table I]

If the vectorlike quarks are stable, then they form QCD bound states. The lightest of these is a heavy-light meson involving a vectorlike quark and a $u$ or $d$ quark. If this heavy-light meson is electrically neutral, then it can be a component of dark matter. The heavy-light meson, how- ever, interacts with nucleons (e.g., via meson exchange) and also has a magnetic dipole moment, so that there are stringent limits on the mass of the vectorlike quark from direct searches for dark matter.

A simpler alternative is that all vectorlike quarks decay into SM particles. For that to happen, the charge $z$ must take certain values, or else there must be additional scalars with VEVs carrying $U(1)_{B}$ charge.

In the simplest case, the $D=U$ model with $n=$ 1 [31], we find that $z=1 / 3$ would allow decays into a SM quark and $h^{0}$ (the SM Higgs boson) through the Yukawa interactions

$$
\bar{q}_{L} U_{R} H, \bar{q}_{L} D_{R} H^{\dagger}, \bar{u}_{R} Q_{L} H^{\dagger}, \bar{d}_{R} Q_{L} H,
$$

where $H$ is the SM Higgs doublet. In addition, decays into a SM quark and $\phi$ may proceed through the following Yukawa terms:

$$
\bar{q}_{L} Q_{R} \phi^{\dagger}, \bar{u}_{R} U_{L} \phi^{\dagger}, \bar{d}_{R} D_{L} \phi^{\dagger}
$$

Even when the two particles described by the complex scalar $\phi$ are heavier than the vectorlike quarks, the above Yukawa terms in conjunction with the Higgs portal coupling $H^{\dagger} H \phi^{\dagger} \phi$ induce $Q, U$ and $D$ decays, of the type $Q \rightarrow q h^{0} h^{0}$ (or $Q \rightarrow q b \bar{b} h^{0}$ for masses below $2 M_{h}$ ). For $z=-5 / 3$, the vectorlike quarks decay through Yukawa couplings of the type $\bar{q}_{L} Q_{R} \phi$.

The $D=7 U+3$ model with $n=1$ has different decay patterns. For example, $z=-2 / 3$ implies that the decays $Q_{R} \rightarrow q_{L} \phi$ and $D_{L} \rightarrow d_{R} \phi^{\dagger}$ are 
allowed, but $U$ can decay via renormalizable interactions only if there is at least one additional field (e.g., a scalar $S$ which is a SM gauge singlet, has $U(1)_{B}$ charge 0 , and interacts through $\left.\bar{u}_{R} U_{L} S\right)$.

The $D=U$ and $D=7 U+3$ models are identical for $z=-1 /(2 n)$. In this case, a second scalar $\phi^{\prime}$, of $U(1)_{B}$ charge $1 / 3-1 /(2 n)$, is necessary to allow $Q, U$, and $D$ decays through $\bar{q}_{L} Q_{R} \phi^{\prime}$, $\bar{u}_{R} U_{L} \phi^{\prime}$ and $\bar{d}_{R} D_{L} \phi^{\prime}$, respectively.

The choice of vectorlike fermions shown in Table I is simple but not unique. For example, anomaly cancellation in the presence of vectorlike leptons instead of quarks is also possible [38]. A fourth generation of chiral quarks and leptons can also lead to the cancellation of the $U(1)_{B}$ anomalies [39], but this possibility is nearly ruled out [36] now by the measurements of Higgs production through gluon fusion [40], and by direct searches for $t^{\prime}$ [41] and $b^{\prime}$ [42] quarks at the LHC.

The couplings of the $Z_{B}^{\prime}$ to SM quarks are given by

$$
\frac{g_{B}}{6} Z_{B \mu}^{\prime} \bar{q} \gamma^{\mu} q
$$

where $g_{B}$ is the $U(1)_{B}$ gauge coupling (using the normalization where the group generator is $1 / 2$ ), and is related to the coupling constant, as usual, by $\alpha_{B}=g_{B}^{2} /(4 \pi)$. The $Z_{B}^{\prime}$ can decay into a pair of jets (including $b$ jets) or into a $t \bar{t}$ pair (for a $Z_{B}^{\prime}$ mass $M_{Z_{B}^{\prime}}>2 m_{t}$ ), with partial decay widths given by

$$
\begin{aligned}
& \Gamma\left(Z_{B}^{\prime} \rightarrow j j\right)=\frac{5 \alpha_{B}}{36} M_{Z_{B}^{\prime}}\left(1+\frac{\alpha_{s}}{\pi}\right) \\
& \frac{\Gamma\left(Z_{B}^{\prime} \rightarrow t \bar{t}\right)}{\Gamma\left(Z_{B}^{\prime} \rightarrow j j\right)}=\frac{1}{5}\left(1-\frac{4 m_{t}^{2}}{M_{Z_{B}^{\prime}}^{2}}\right)^{1 / 2}\left[1+O\left(\frac{\alpha_{s} m_{t}}{M_{Z_{B}^{\prime}}}\right)\right]
\end{aligned}
$$

Here we have included the NLO QCD corrections and no electroweak corrections. If the decays into vectorlike quarks are kinematically closed, then the total width of $Z_{B}^{\prime}$ is

$$
\Gamma_{Z_{B}^{\prime}}=\Gamma\left(Z_{B}^{\prime} \rightarrow j j\right)+\Gamma\left(Z_{B}^{\prime} \rightarrow t \bar{t}\right) .
$$

\section{B. Coloron}

Another hypothetical particle that can easily produce dijet resonances with large cross section at the LHC is the coloron [32], a spin-1 coloroctet gauge boson. The coloron, in the case of flavor-universal couplings [33], is not significantly constrained by flavor processes nor by other low energy data. Furthermore, the coloron is automatically leptophobic.

The simplest gauge symmetry that can be associated with a heavy color-octet vector boson is $S U(3)_{1} \times S U(3)_{2}$ [43]. This is spontaneously broken down to the diagonal $S U(3)_{c}$ gauge group, which is identified with the QCD one. A minimal renormalizable extension of the SM which includes a coloron, dubbed ReCoM, is analyzed in Ref. [34]. Assuming that all the SM quarks transform as $(3,1)$ under $S U(3)_{1} \times S U(3)_{2}$, the couplings of the coloron to SM quarks are given 
by the Lagrangian term

$$
g_{s} \tan \theta \bar{q} \gamma^{\mu} T^{a} G_{\mu}^{\prime a} q
$$

where $g_{s}=\sqrt{4 \pi \alpha_{s}}$ is the QCD gauge coupling and $\tan \theta>0$ is a dimensionless parameter.

If there are no new quarks mixing with the SM ones, and no additional color-octet spin-1 particles, then $\tan \theta$ is the ratio of the $S U(3)_{2}$ and $S U(3)_{1}$ gauge couplings. These gauge couplings can vary between $g_{s}$ and some upper limit of about $4 \pi / \sqrt{3}$ corresponding to the nonperturbative regime. Consequently, there are both upper and lower limits on $\tan \theta$ [44]: $0.15 \lesssim \tan \theta \lesssim$ 6.7. Unlike the $Z_{B}^{\prime}$, whose UV behavior requires some new fermions, the flavor-universal coloron is anomaly free. Nevertheless, vectorlike quarks may be present, and if they mix with the SM quarks, then the lower limit on $\tan \theta$ no longer applies [45]. Similarly, a second heavy spin-1 color-octet particle can mix with the coloron and dilute its couplings to quarks.

The partial decay widths of the coloron of mass $M_{G^{\prime}}$ into $j j$ (including $b \bar{b}$ ) and into $t \bar{t}$ are given by

$\Gamma\left(G^{\prime} \rightarrow j j\right)=\frac{5 \alpha_{s}}{6} \tan ^{2} \theta M_{G^{\prime}}\left[1+O\left(\frac{\alpha_{s}}{\pi}\right)\right]$,

$\frac{\Gamma\left(G^{\prime} \rightarrow t \bar{t}\right)}{\Gamma\left(G^{\prime} \rightarrow j j\right)}=\frac{1}{5}\left(1-\frac{4 m_{t}^{2}}{M_{G^{\prime}}^{2}}\right)^{1 / 2}\left[1+O\left(\frac{\alpha_{s} m_{t}}{M_{G^{\prime}}}\right)\right]$, where only NLO QCD corrections are included.

The minimal scalar sector responsible for breaking the $S U(3)_{1} \times S U(3)_{2}$ symmetry (which is part of ReCoM) includes a color octet and two color singlets. If these are light enough, then the coloron can decay into two octet scalars or into an octet scalar and a singlet scalar, with partial decay widths that are especially large for $\tan \theta \ll 1$ [34]. In what follows, we will assume that the scalars are heavier than $M_{G^{\prime}} / 2$, so that the total width of the coloron is simply the sum of the $j j$ and $t \bar{t}$ partial widths shown in Eq. (10).

\section{COLLIDER SEARCHES OF DIJET RESONANCES}

We now detail our procedure and results for mapping the existing dijet resonance searches to the coupling-mass plane.

\section{A. Mapping procedure and experimental limits}

As discussed in Section I, the partons responsible for $s$-channel production at hadron colliders are also a decay mode, and so the new particle must decay back to pairs of jets at some rate. Models that give rise to a spin-1 dijet resonance are the most straightforward to construct. For the representative spin-1 particles discussed in Section III the $Z_{B}^{\prime}$ boson and the coloron, there are two parameters that characterize the dijet signal: mass and coupling.

Even with only two parameters, the extraction of limits from experimental searches for dijet resonances remains challenging. For example, varying the resonance mass while keeping the coupling fixed introduces varying levels of 
final state radiation, cut-dependent effects from parton distribution function (PDF) sampling at high masses relative to the total $\sqrt{s}$ (the mass dependence of PDFs is shown in [46]), and triggerdependent efficiencies at low masses.

Dijet resonance searches probe the existence of narrow peaks in the dijet invariant mass $\left(m_{j j}\right)$ spectrum. The QCD background is expected to be a smoothly falling exponential. Other backgrounds, such as hadronic $t \bar{t}$ decays, are expected to give broad features at their respective mass scales.

Although a bump-like feature on top of a smoothly falling background is seemingly easy to observe, the experimental resolution in the dijet channel is rather poor $(\sim 5-10 \%$, depending on mass 2] as well as experiment), and the QCD background at energies much smaller than the total $\sqrt{s}$ become overwhelming. Higher $\sqrt{s}$ colliders rapidly lose sensitivity to low mass resonances in dijet searches because of the minimum $p_{T}, E_{T}$, and $m_{j j}$ trigger requirements. Prescaled triggers (and so-called "data-scouting" techniques [23, 47]), however, can help augment the trigger bandwidth to extend the searches down to lower masses.

For our mapping, we start by running a Monte Carlo (MC) simulation for a given choice of coupling and mass. In the narrow width approximation, the $s$-channel production factorizes from the decay, hence the acceptance and efficiency do not depend on the coupling at leading order. Some dependence on the coupling arises from loops involving the new spin-1 particle, as shown in the case of NLO coloron production [48]; however, this effect is relatively small, and for simplicity we ignore it in what follows. For a given set of experimental cuts we obtain a simulated effective rate. The ratio of the experimental limit to the simulated effective rate is the square of a coupling rescaling factor. Multiplying the initial coupling by this rescaling factor gives the experimental upper limit on the coupling for the chosen mass. Repeating this procedure for all experimental searches, we determine the excluded region in the coupling-mass plane.

There has been a host of resonance searches from every experiment at hadron colliders in the dijet channel. We summarize all of them in Table II.

For each mass point and collider, we simulate an event sample of on-shell $s$-channel $Z_{B}^{\prime}$ as well as coloron production (at leading order) with subsequent decay to light-flavor and $b$ jets using MadGraph 5 v1.5.7 [49] with the CTEQ6L1 PDFs [50]. Each event is passed through PYTHIA v6.4.20 [51] for showering and hadronization, and then through PGS v4 [52] for basic detector simulation and jet clustering.

Choosing $g_{B}=0.2$ for the $Z_{B}^{\prime}$ or $\tan \theta=0.2$ for the coloron, we obtain the cross section times branching fraction from MadGraph 5, denoted $\sigma_{0.2} \cdot B$, as a function of mass for each collider. We then implement the various triggers and cuts 


\begin{tabular}{|c|c|c|c|c|}
\hline $\begin{array}{l}\text { Collisions, } \\
\sqrt{s}(\mathrm{TeV})\end{array}$ & Experiment & Ref. & $\begin{array}{c}\text { Luminosity } \\
\left(\mathrm{fb}^{-1}\right)\end{array}$ & $\begin{array}{c}\text { Mass range } \\
(\mathrm{TeV})\end{array}$ \\
\hline \multirow[t]{2}{*}{$p \bar{p}, 0.63$} & UA2 & $\begin{array}{l}3] \\
{[4]}\end{array}$ & $\begin{array}{l}4.7 \times 10^{-3} \\
1.1 \times 10^{-2}\end{array}$ & $\begin{array}{l}0.08-0.32 \\
0.14-0.3\end{array}$ \\
\hline & UA1 & [5] & $4.9 \times 10^{-4}$ & $0.15-0.4$ \\
\hline \multirow[t]{2}{*}{$p \bar{p}, 1.8$} & $\mathrm{CDF}$ & \begin{tabular}{|l}
{$[6]$} \\
{$[7]$} \\
{$[8]$} \\
{$[9]$}
\end{tabular} & $\begin{array}{c}2.6 \times 10^{-6} \\
4.2 \times 10^{-3} \\
1.9 \times 10^{-2} \\
0.11\end{array}$ & $\begin{array}{c}0.06-0.5 \\
0.2-0.9 \\
0.2-1.15 \\
0.2-1.15\end{array}$ \\
\hline & D0 & [11] & 0.11 & $0.2-0.9$ \\
\hline$p \bar{p}, 1.96$ & $\mathrm{CDF}$ & [10] & 1.1 & $0.26-1.4$ \\
\hline \multirow[t]{2}{*}{$p p, 7$} & ATLAS & $\begin{array}{l}12] \\
{[13]} \\
{[14]} \\
{[15]} \\
{[16]} \\
{[17]}\end{array}$ & $\begin{array}{c}3.2 \times 10^{-4} \\
3.6 \times 10^{-2} \\
0.16 \\
0.81 \\
1.0 \\
4.8\end{array}$ & $\begin{array}{l}0.3-1.7 \\
0.6-4 \\
0.9-4 \\
0.9-4 \\
0.9-4 \\
1-4\end{array}$ \\
\hline & CMS & $\begin{array}{l}{[20]} \\
{[21]} \\
{[22]} \\
{[23]} \\
\end{array}$ & $\begin{array}{c}2.9 \times 10^{-3} \\
1.0 \\
5.0 \\
0.13 \\
\end{array}$ & $\begin{array}{c}0.5-2.6 \\
1-4.1 \\
1-4.3 \\
0.6-1 \\
\end{array}$ \\
\hline \multirow[t]{2}{*}{$p p, 8$} & ATLAS & $\begin{array}{l}{[18]} \\
{[19]} \\
\end{array}$ & $\begin{array}{l}5.8 \\
13 \\
\end{array}$ & $\begin{array}{l}1.5-4 \\
1.5-4.8\end{array}$ \\
\hline & CMS & $\begin{array}{l}{[24]} \\
{[25]} \\
\end{array}$ & $\begin{array}{l}4.0 \\
20\end{array}$ & $\begin{array}{r}1-4.8 \\
1.2-5.3\end{array}$ \\
\hline
\end{tabular}

TABLE II. Mass ranges for existing dijet resonance searches at hadron colliders.

as described in each analysis listed in Table II, to obtain an acceptance $A$. The ratio of the resulting simulated effective rate, $\sigma_{0.2} \cdot B \cdot A$, to the limit from each relevant analysis in Table II allows us to extract the upper limit on the coupling as a function of mass:

$$
\left(g_{B}\right)_{\max }=0.2\left(\frac{(\sigma \cdot B \cdot A)_{\text {limit }}}{\sigma_{0.2} \cdot B \cdot A}\right)^{1 / 2},
$$

and similarly for $(\tan \theta)_{\max }$.

We now discuss the most relevant searches, grouped according to the mass range probed.

\section{Searches for $m_{j j}<200 \mathrm{GeV}$}

Although a couple of searches (from UA2 and CDF, see Table II) extend to masses below 140 $\mathrm{GeV}$, we do not use them because those results were based on a subtraction of the expected $W$ and $Z$ dijet distribution calculated at $O\left(\alpha_{s}\right)$; modern precision of $W$ and $Z$ two-dijet distributions far exceeds the interpretative power of the effective rate limit in the $60-140 \mathrm{GeV}$ mass window. For studies of theoretical constraints on colorons in that mass range, see Ref. [53].

In the $140-200 \mathrm{GeV}$ mass range, by far the largest data sample $\left(10.9 \mathrm{pb}^{-1}\right)$ has been analyzed [4] by the UA2 experiment at the CERN SPS collider, which operated mainly at $\sqrt{s}=630$ GeV. Mapping the UA2 limit to the couplingmass plane is simpler than the procedure required for later analyses because the UA2 analysis includes a table of efficiencies for selecting the signal events from background as well as for isolating the peak feature in the signal events (cf. Table 1 and Table 2 of Ref. [4]). We linearly interpolate this overall efficiency to obtain a combined acceptance times efficiency factor. Finally, since the UA2 constraint is presented as a branching 
fraction limit on a sequential SM $Z^{\prime}$, we unfold the $Z^{\prime}$ cross section to obtain an estimated $\sigma \cdot B$ limit, as discussed in Ref. [54]. After applying the overall efficiency, we obtain an effective rate limit from UA2, which we then map into the upper limit on the $Z_{B}^{\prime}$ coupling shown on the left-hand side of Figure 1 .

2. Searches in the 200 - 900 GeV mass range

The CDF [9] and D0 [11] searches using the full data samples $\left(\approx 110 \mathrm{pb}^{-1}\right)$ of the Run I at the Tevatron compete for the best limit in the $200-$ $260 \mathrm{GeV}$ mass window. We choose to extract a limit from the CDF analysis, because it applies to a larger mass range.

Above $260 \mathrm{GeV}$, the $\mathrm{CDF}$ analysis using 1.1 $\mathrm{fb}^{-1}$ of Run II data [10] supersedes the Run I results. For the $260-900 \mathrm{GeV}$ window, the only ATLAS [12, 13] and CMS [20, 23] searches use very small $\sqrt{s}=7 \mathrm{TeV}$ data sets. The most competitive of these is the $0.13 \mathrm{fb}^{-1} \mathrm{CMS}$ [23] analysis via so-called "data scouting", which uses a reduced data format to record events with sensitivity at low masses even during high instantaneous luminosity conditions. We implement the appropriate trigger and analysis requirements for each mass point probed by these CDF and CMS analyses to calculate their respective acceptances needed for the coupling-mass mapping. For all CMS limits, we adopt their $q q$ resonance constraint, since our spin-1 resonances only couple (at leading order) to $q \bar{q}$ (note that the radiation patterns from $q q$ and $q \bar{q}$ final states are indistinguishable). As can be seen from Figure 1, the CDF limits on spin-1 resonances are the most stringent ones, even though they are based on only a tenth of the Run II data.

\section{Searches for resonance masses above $900 \mathrm{GeV}$}

Most ATLAS and CMS searches begin at about $900 \mathrm{GeV}$. For the $900-1000 \mathrm{GeV}$ range, the ATLAS $1 \mathrm{fb}^{-1}$ search [16] is expected to be the most sensitive, as it has higher energy than CDF $1.1 \mathrm{fb}^{-1}$ [10], and a larger data sample than the other ATLAS [12 15] and CMS studies [20, 23].

From $1-1.2 \mathrm{TeV}$, the CMS $4.0 \mathrm{fb}^{-1}$ search using $8 \mathrm{TeV}$ data [24] is expected to be competitive with the earlier $7 \mathrm{TeV}$ ATLAS $4.8 \mathrm{fb}^{-1}$ [17] and CMS $5.0 \mathrm{fb}^{-1}$ analyses [22], superseding the ATLAS and CMS $1 \mathrm{fb}^{-1}$ analyses [16, 21]. The slightly smaller amount of integrated luminosity analyzed in Ref. [24] compared to Refs. [17, 22] is counterbalanced by the slight increase in collider energy, giving comparable coupling sensitivities.

Above 1.2 TeV, the CMS analysis [25] using $19.6 \mathrm{fb}^{-1}$ of $\sqrt{s}=8 \mathrm{TeV}$ data is expected to be the most sensitive (we will refer to this search as CMS $\left.20 \mathrm{fb}^{-1}\right)$. This analysis is the most recent dijet resonance search and has sensitivity to resonances as heavy as $5 \mathrm{TeV}$. Nevertheless, upward fluctuations in the CMS $20 \mathrm{fb}^{-1}$ limit actually 
leave some small gaps where the ATLAS [19] 13.0 $\mathrm{fb}^{-1}$ limit and the CMS $5.0 \mathrm{fb}^{-1}$ limits are more stringent (see Figures 1 and 21).

For the various CMS analyses, we implement the respective trigger and analysis cuts to tabulate the acceptance for each mass point and obtain an effective rate limit from the $q q$ resonance constraint. For the $8 \mathrm{TeV}$ analyses we find that the acceptance grows from $38 \%$ to $50 \%$ for the $Z_{B}^{\prime}$ signal (and from $33 \%$ to $44 \%$ for the coloron) when the mass grows from $1 \mathrm{TeV}$ to $2.5 \mathrm{TeV}$, and is constant at larger masses.

The ATLAS results, on the other hand, are presented as limits on Gaussian resonances in the $m_{j j}$ spectrum after trigger requirements, detector effects, and analysis cuts are implemented. This poses additional problems because the $m_{j j}$ distribution produced by any particle decaying to a pair of jets would be quite different from a Gaussian: a long tail at low invariant mass is induced by imperfect recapturing of final state radiation. To overcome this mismatch, we form the $m_{j j}$ spectrum after cuts and then model and apply a Gaussian core efficiency $\left(\epsilon_{G}\right)$ for our signal, which is an additional factor beyond the canonical acceptance. Our procedure of determining $\epsilon_{G}$ is described in the Appendix.

The ATLAS limits are given for a variety of Gaussian widths: for each ATLAS limit, we adopt the smallest Gaussian width constraint in performing our mapping, since our resonances typically have intrinsic widths at the percent and sub-percent level.

\section{B. Results and Discussion}

Following the procedure described in Section IIA for the leading experimental dijet limits, we obtain the coupling-mass mapping shown in Figure 1 for a spin-1 resonance $Z_{B}^{\prime}$. We have used the leading order production computed with MadGraph 5.

If the NLO corrections to the process $p p \rightarrow$ $Z_{B}^{\prime} X \rightarrow j j X$ are included in an event generator, then the mapping can be performed more precisely. We do not expect that they would change the values of $g_{B}$ by more than $O(10 \%)$.

We emphasize that, unlike the usual $\sigma \cdot B \cdot A$ limit plots, Figure 1 readily shows the exclusion in coupling as well as mass. This mapping also demonstrates the complicated interplay between different experimental analyses using different colliders and luminosities.

From Figure 1, we conclude that $Z_{B}^{\prime}$ bosons are unconstrained for a gauge coupling $g_{B} \lesssim 0.6$, leaving a large area of parameter space unexplored by dijet resonance searches so far. Moreover, for the entire sub-TeV region, the experimental limits allow for $g_{B}$ couplings as large as 0.9 , while locally, $g_{B}$ couplings can reach $\approx 1.5$. Importantly, an update from CDF or an analysis by D0 with their full $\approx 10 \mathrm{fb}^{-1}$ Run II datasets could offer evidence for or provide interesting limits on new sub-TeV dijet resonances. We also 


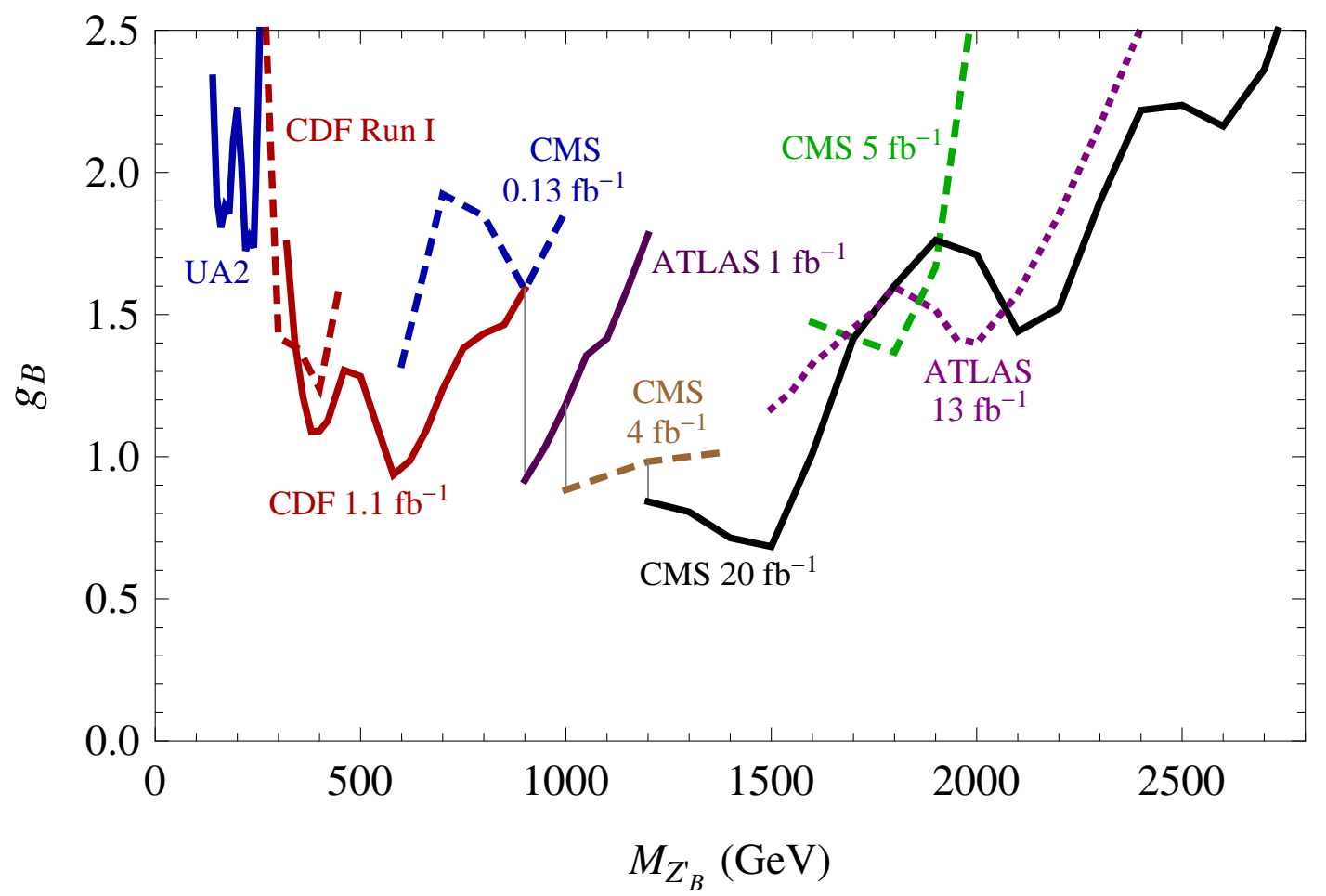

FIG. 1. Leading experimental limits in the coupling $g_{B}$ versus mass $M_{Z_{B}^{\prime}}$ plane for $Z_{B}^{\prime}$ resonances. Values of $g_{B}$ above each line are excluded at the $95 \%$ C.L.

note that an update of the "scouted data" analysis [23] with more luminosity by CMS (and ATLAS) would also push sensitivity to lower couplings in the several hundred $\mathrm{GeV}$ mass range.

The plot is not extended above $g_{B}=2.5$, because the $U(1)_{B}$ coupling constant is already large, $\alpha_{B}=g_{B}^{2} /(4 \pi) \approx 0.5$, so that it is difficult to avoid a Landau pole. For that large coupling, the current mass reach is around $2.8 \mathrm{TeV}$. The $14 \mathrm{TeV}$ LHC will extend significantly the mass reach, and can probe smaller couplings once enough data is analyzed. Note that couplings of $g_{B} \approx 0.1$ can be viewed as typical (the analogous coupling of the photon is approximately 0.3 ), and even $g_{B}$ as small as 0.01 would not be very surprising.

We also present the coupling-mass mapping for colorons in Figure 2, For clarity, we only show the envelope of the strongest $\tan \theta$ upper limits from all available analyses at each coloron mass. This mapping is performed again using leading order production. The NLO corrections to coloron production have been computed recently [48], and can vary between roughly $-30 \%$ and $+20 \%$. We do not take the NLO corrections into account as we do not have an event generator that includes them; furthermore, there is some model dependence in the NLO corrections 


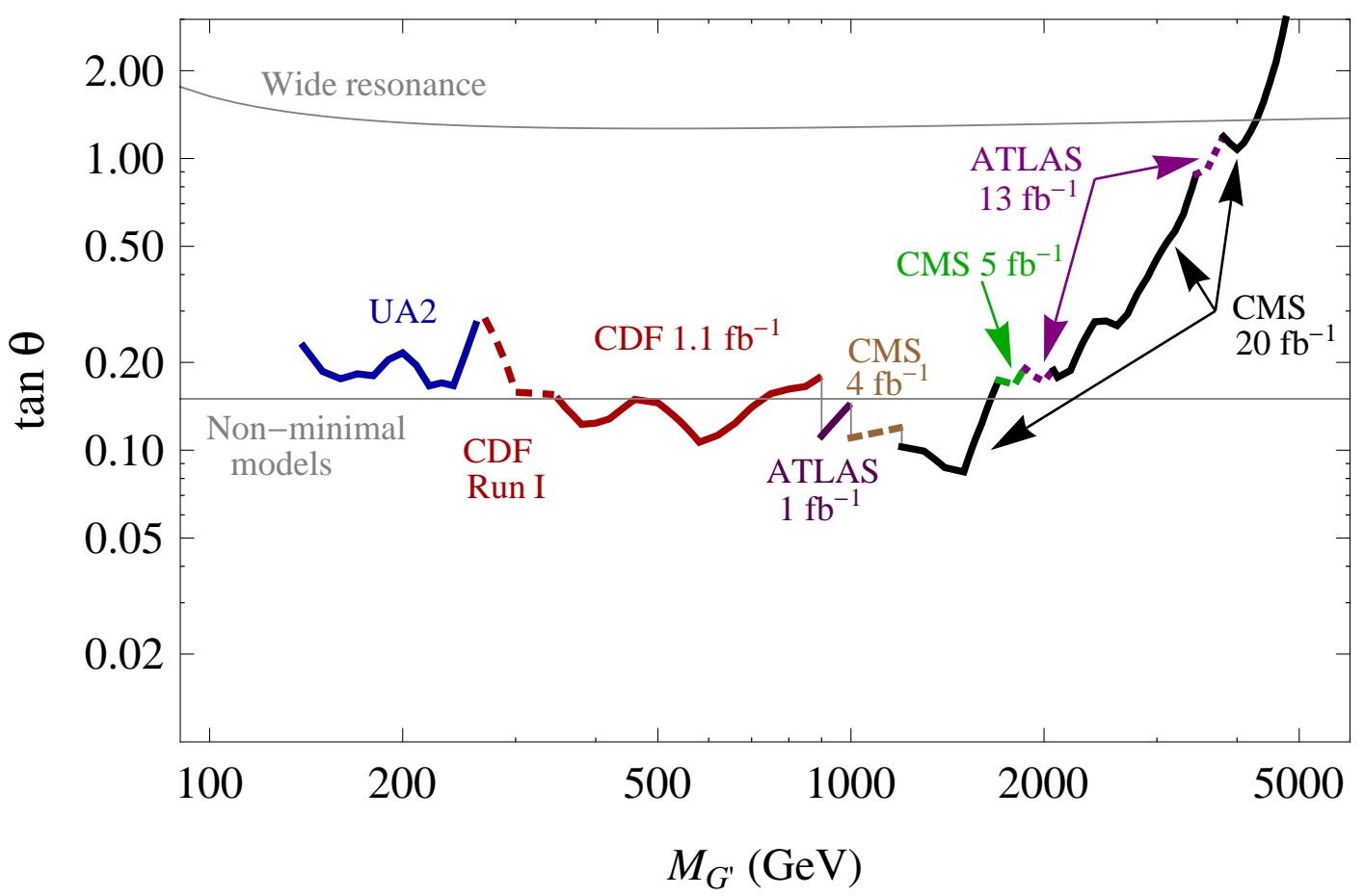

FIG. 2. Envelope of experimental limits for colorons of coupling $\tan \theta$ and mass $M_{G^{\prime}}$. Values of $\tan \theta$ above each limit are excluded at the $95 \%$ C.L. The region above the line labelled "Wide resonance" corresponds to a coloron width-to-mass ratio larger than $15 \%$. The region below the line labelled "Non-minimal models" ( $\operatorname{at} \tan \theta=0.15$ ) requires additional particles, such as vectorlike quarks or a second coloron.

at $\operatorname{small} \tan \theta$ (for example, they are sensitive to the color-octet scalar present in ReCoM [34]).

As mentioned in Section $\llbracket$ IIB, in the minimal coloron model there is a theoretical lower limit imposed by perturbativity, $\tan \theta \gtrsim 0.15$. The experimental upper limit dips below that value only in the $350-700 \mathrm{GeV}$ and $0.9-1.6 \mathrm{TeV}$ mass ranges. In non-minimal models, where there are vectorlike quarks or a second coloron, $\tan \theta$ can be substantially smaller than 0.15. Thus, searches for colorons should continue even after they rule out $\tan \theta \gtrsim 0.15$ in some mass windows.
On the contrary, the discovery of a coloron with $\tan \theta<0.15$ would imply the existence of additional colored particles that can be probed in hadron collisions.

Unlike the $U(1)$ gauge bosons, the coloron can be rather strongly coupled before reaching the perturbative upper limit, $\tan \theta \lesssim 6.7$, because it is associated with a non-Abelian gauge interaction that is asymptotically free.

There is, however, a tighter upper limit on $\tan \theta$ if the total width, $\Gamma\left(G^{\prime} \rightarrow j j\right)+\Gamma\left(G^{\prime} \rightarrow t \bar{t}\right)$ [see Eq. (10)], is required to be smaller than 
the dijet resolution. A ratio of the total width to $M_{G^{\prime}}$ of $15 \%$ (as used in [16]) corresponds to $\tan \theta \approx 1.3$ for $M_{G^{\prime}} \gg 2 m_{t}$ (with a mild dependence on $M_{G^{\prime}}$ due to the running of $\alpha_{s}$ ), and to slightly larger $\tan \theta$ for smaller masses, as shown in Figure 2. Limits above the line marked there by "Wide resonance" are not reliable if set by narrow resonance searches [55]. Clearly, resonances that have a much broader intrinsic width than the experimental $m_{j j}$ resolution will fade more easily into the exponentially falling QCD background. Separately, for large enough coupling, the $t$-channel exchange of a coloron starts contributing significantly to the dijet signal, further diluting the $m_{j j}$ peak. Note also that at resonance masses approaching the total $\sqrt{s}$ of the collider, PDF uncertainties increase.

\section{CONCLUSIONS}

We urge the experimental collaborations to present limits (or contours if a signal is observed) on dijet resonances in the coupling-versus-mass plane of a "baryonic" $Z_{B}^{\prime}$, as in Figure 1 (or a coloron as in Figure 2, if the search is sensitive primarily to large signals arising from heavy resonances). This coupling-mass mapping, while being somewhat model dependent (and thus a complement to - not a replacement for-the crosssection limit plots), has multiple advantages. First, it allows a comparison of limits set by experiments performed at different colliders, and at different center-of-mass energies. Second, it allows an assessment of how stringent the experimental limits are, by comparing them with the expected range of the physical coupling. Third, it provides a direct interpretation, without the need for $\mathrm{MC}$ simulations to compute the acceptance or to convert limits on a Gaussian into limits on a realistic particle (in the case of the existing ATLAS results).

The coupling-mass mapping also highlights gaps in the combined sensitivity of all existing searches. Figure 1 shows that the coupling reach is rather poor in the mass range of $700-900 \mathrm{GeV}$, and it is even worse for masses below about 300 GeV. A new analysis by CDF or D0 with the full Run II data set could have great impact there. Non-conventional methods, such as analyzing scouted data [23], are also important for extending the sensitivity of LHC experiments in the sub-TeV mass range. More generally, the traditional trend for each new dijet search to attain ever higher mass reach does not need to leave the (equally important) small-coupling region unexplored.

We have argued that the simplest origin of narrow dijet resonances is a spin-1 particle with flavor-independent couplings. Our mapping thus focused on the $Z_{B}^{\prime}$ and $G^{\prime}$. The same procedure can also be applied to other spins or color representations [1], but the results would be different because of PDF dependencies and radiation patterns of the decay products. 
The overview of theoretical and experimental status of dijet resonances included in this paper is not exhaustive. For example, we have not discussed angular correlations, which complement the information contained in the $m_{j j}$ distribution. We also note that any particle that produces a dijet resonance can also be produced in association with a $W$, a $Z$ or a photon. Even though the cross sections for these associated productions are much smaller [34, 56], the searches for $W+j j, Z+j j$, and $\gamma+j j$ benefit from better triggers that extend sensitivity to lower resonance masses compared to the pure dijet resonance searches.

The coupling-mass plane can and should be used for any resonance search (as it has been done in some cases, e.g., [8, 9, [57, 58]). In particular, the $t \bar{t}$ resonance searches can be interpreted in terms of the same $Z_{B}^{\prime}$ or coloron. For these flavor-blind particles, it would also be interesting to investigate the complementarity between $t \bar{t}$ and dijet resonance searches.

If a dijet resonance will be discovered in the absence of a dilepton resonance at the same mass, it is likely that additional colored particles will remain to be discovered. To see this, recall (from Section (II) that the $Z_{B}^{\prime}$ requires some vectorlike fermions to cancel the gauge anomalies, while the coloron requires at least some scalars from the gauge symmetry breaking sector.

\section{ACKNOWLEDGEMENTS}

We would like to thank Sekhar Chivukula, Arsham Farzinnia, Robert Harris, Olivier Mattelaer, Elizabeth Simmons and Ciaran Williams for useful communications. We are grateful to James Bourbeau for pointing out an error in an earlier version of Eq. (3). Fermilab is operated by the Fermi Research Alliance, LLC under Contract No. De-AC02-07CH11359 with the United States Department of Energy.

\section{APPENDIX: FROM GAUSSIANS TO PARTICLES}

As explained in Section IIA, the effective rate in the case of ATLAS analyses is given by $\sigma \cdot B \cdot A \cdot \epsilon_{G}$, where $\epsilon_{G}$ is the efficiency of converting the limits on a realistic particle (whose $m_{j j}$ distribution has a long tail due to final state radiation) into limits on a Gaussian. As noted in the Appendix of Ref. [16], the low- $m_{j j}$ tail should be removed as it does not contribute to the assumed Gaussian signal. In this Appendix we present a more precise procedure for estimating $\epsilon_{G}$.

We fit the $m_{i j}$ signal spectrum with a Crystal Ball function [59],

$$
\begin{aligned}
& f(x ; \alpha, n, \bar{x}, \sigma)=N \\
& \times\left\{\begin{array}{c}
\exp \left(-\frac{(x-\bar{x})^{2}}{2 \sigma^{2}}\right), \text { for } \frac{x-\bar{x}}{\sigma}>-\alpha, \\
A\left(\frac{n}{|\alpha|}-|\alpha|-\frac{x-\bar{x}}{\sigma}\right)^{-n}, \text { for } \frac{x-\bar{x}}{\sigma} \leq-\alpha,
\end{array}\right.
\end{aligned}
$$

which is a combination of a truncated Gaussian 


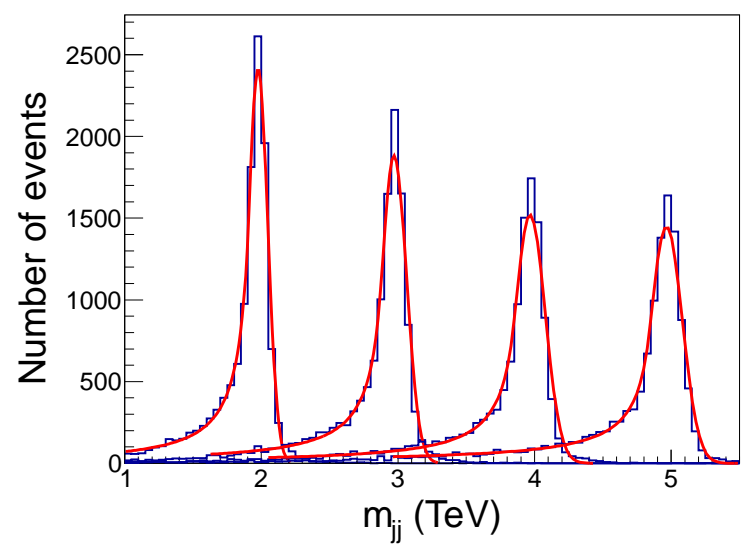

FIG. 3. Simulated $m_{j j}$ distributions (blue histograms) and Crystal Ball fits (red curves) performed on $25000 Z^{\prime}$ signal events for $M_{Z_{B}^{\prime}}=2,3,4$, and 5 $\mathrm{TeV}$, after implementing the CMS $20 \mathrm{fb}^{-1}$ search [25].

and a power law tail; here

$$
A=\left(\frac{n}{|\alpha|}\right)^{n} e^{-|\alpha|^{2} / 2}
$$

and $N$ is an overall normalization factor. The fit parameters $\alpha, n, \bar{x}$, and $\sigma$ correspond to the location of the power law-Gaussian crossover in units of $\sigma$, the power law exponent, and the mean and width of the Gaussian, respectively. Performing this fit allows us to use the Gaussian fit parameters to calculate the Gaussian core efficiency.

We have checked that this fitting function accurately reproduces the expected signal shape for our on-shell $s$-channel resonance production in the $m_{j j}$ spectrum, as shown in Figure 3, which is in reasonable agreement with Fig. 2 of Ref. [25] ]. The fit parameters are varied within a large range of values: $\alpha$ floats within 0.05 to $3.5, n$ within 0.5 to $5.0, \bar{x}$ within $(70-105) \%$ of the truth boson mass, $\sigma$ within $(0.5-30) \%$ of the truth boson mass, and the overall normalization $N$ floats within (50-300)\% of the $N_{\text {events }}^{\text {total }}$ in the histogram. Moreover, the beginning and end of the fit range is adjusted from $(50-70) \%$ and $(110-115) \%$ of the $m_{j j}$ peak, respectively. The fit range with the smallest $\chi^{2} /$ ndf dictates the fit parameters $\bar{x}$ and $\sigma$ used in the estimation of the Gaussian core component of the $m_{j j}$ shape.

From the fit parameters, we count the number of events in the $m_{j j}$ distribution within $\bar{x} \pm 3 \sigma$ to give the efficiency $\epsilon_{G}$ for extracting the Gaussian peak appropriate for the Gaussian template limits set by ATLAS,

$$
\epsilon_{G}=\frac{N_{\text {events }}^{\bar{x} \pm 3 \sigma}}{N_{\text {events }}^{\text {total }}}
$$

From our simulated ATLAS $m_{j j}$ distributions, we get $\epsilon_{G}$ values for both the $Z_{B}^{\prime}$ and the coloron between $55 \%$ and $65 \%$.

\footnotetext{
${ }^{1}$ Even though we only use the Gaussian fit procedure on ATLAS limits, we show the fit results from our implementation of the CMS $20 \mathrm{fb}^{-1}$ study to facilitate comparison with Fig. 2 of Ref. [25]. The corresponding $m_{j j}$ spectrum and Crystal Ball fits for ATLAS analyses are
}

[1] T. Han, I. Lewis and Z. Liu, "Colored resonant signals at the LHC: largest rate and simplest topology," JHEP 1012, 085 (2010)

qualitatively similar. 


\section{arXiv:1010.4309.}

[2] R. M. Harris and K. Kousouris, "Searches for Dijet Resonances at Hadron Colliders," Int. J. Mod. Phys. A 26, 5005 (2011) arXiv:1110.5302.

[3] J. Alitti et al. [UA2 Collaboration], "A measurement of two jet decays of the $W$ and $Z$ bosons at the CERN $\bar{p} p$ collider", Z. Phys. C 49, 17 (1991).

[4] J. Alitti et al. [UA2 Collaboration], "A search for new intermediate vector mesons and excited quarks decaying to two jets at the CERN $\bar{p} p$ collider", Nucl. Phys. B 400, 3 (1993).

[5] C. Albajar et al. [UA1 Collaboration], "Two jet mass distributions at the CERN proton - antiproton collider," Phys. Lett. B 209, 127 (1988).

[6] F. Abe et al. [CDF Collaboration], "The two jet invariant mass distribution at $\sqrt{s}=1.8 \mathrm{TeV}$," Phys. Rev. D 41, 1722 (1990).

[7] F. Abe et al. [CDF Collaboration], "Search for quark compositeness, axigluons and heavy particles using the dijet invariant mass spectrum observed in $p \bar{p}$ collisions," Phys. Rev. Lett. 71 (1993) 2542.

[8] F. Abe et al. [CDF Collaboration], "Search for new particles decaying to dijets in $p \bar{p}$ collisions at $\sqrt{s}=1.8 \mathrm{TeV}$," Phys. Rev. Lett. 74, 3538 (1995) hep-ex/9501001.

[9] F. Abe et al. [CDF Collaboration], "Search for new particles decaying to dijets at CDF," Phys. Rev. D 55, 5263 (1997) hep-ex/9702004.

[10] T. Aaltonen et al. [CDF Collaboration], "Search for new particles decaying into dijets in protonantiproton collisions at $\sqrt{s}=1.96 \mathrm{TeV}$," Phys. Rev. D 79, 112002 (2009) arXiv:0812.4036.

[11] V. M. Abazov et al. [D0 Collaboration], "Search for new particles in the two jet decay channel", Phys. Rev. D 69, 111101 (2004) hep-ex/0308033.

[12] G. Aad et al. [ATLAS Collaboration], "Search for new particles in two-jet final states in $7 \mathrm{TeV}$ proton-proton collisions", Phys. Rev. Lett. 105, 161801 (2010) arXiv:1008.2461.

[13] G. Aad et al. [ATLAS Collaboration], "Search for New Physics in Dijet Mass and Angular Distributions in pp Collisions at $\sqrt{s}=7 \mathrm{TeV}$ ", New J. Phys. 13, 053044 (2011) arXiv:1103.3864.

[14] ATLAS Collaboration, "Update of the Search for New Physics in the Dijet Mass Distribution in $163 \mathrm{pb}^{-1}$ of $p p$ Collisions at $\sqrt{s}=7 \mathrm{TeV}$ ", note ATLAS-CONF-2011-081, June 2011.

[15] ATLAS Collaboration, "Search for New Physics in Dijet Mass Distributions in $0.81 \mathrm{fb}^{-1}$ of $p p$ Collisions at $\sqrt{s}=7 \mathrm{TeV}$ ", note ATLAS-CONF2011-095, July 2011.

[16] G. Aad et al. [ATLAS Collaboration], "Search for new physics in the dijet mass distribution using $1 \mathrm{fb}^{-1}$ of $p p$ collision data at $\sqrt{s}=7 \mathrm{TeV}$ ", Phys. Lett. B 708, 37 (2012) arXiv:1108.6311.

[17] G. Aad et al. [ATLAS Collaboration], "Search for new phenomena in dijet mass and angular distributions using $p p$ collisions at $\sqrt{s}=7 \mathrm{TeV}$," JHEP 1301, 029 (2013) arXiv:1210.1718.

[18] ATLAS Collaboration, "Search for New Phenomena in the Dijet Mass Distribution using 5.8 $f b^{-1}$ of $p p$ Collisions at $\sqrt{s}=8 \mathrm{TeV}$, note ATLAS-CONF-2012-088, July 2012.

[19] ATLAS Collaboration, "Search for New Phenomena in the Dijet Mass Distribution updated using $13.0 \mathrm{fb}^{-1}$ of $p p$ Collisions at $\sqrt{s}=8 \mathrm{TeV}$ ", note ATLAS-CONF-2012-148, Nov. 2012.

[20] V. Khachatryan et al. [CMS Collaboration], "Search for Dijet Resonances in $7 \mathrm{TeV}$ pp Collisions at CMS," Phys. Rev. Lett. 105, 211801 
(2010) arXiv:1010.0203 [hep-ex]].

[21] S. Chatrchyan et al. [CMS Collaboration], "Search for Resonances in the Dijet Mass Spectrum from $7 \mathrm{TeV}$ pp Collisions at CMS," Phys. Lett. B 704, 123 (2011) arXiv:1107.4771.

[22] S. Chatrchyan et al. [CMS Collaboration], "Search for narrow resonances and quantum black holes in inclusive and $b$-tagged dijet mass spectra from $p p$ collisions at $\sqrt{s}=7 \mathrm{TeV}$," JHEP 1301, 013 (2013) arXiv:1210.2387.

[23] CMS Collaboration, "Search for narrow resonances using the dijet mass spectrum in pp collisions at $\sqrt{s}=7 \mathrm{TeV}$ ", note CMS-PAS-EXO-11094, July 2012.

[24] S. Chatrchyan et al. [CMS Collaboration], "Search for narrow resonances using the dijet mass spectrum in pp collisions at $\sqrt{s}=8 \mathrm{TeV}$," arXiv:1302.4794 [hep-ex].

[25] CMS Collaboration, "Search for Narrow Resonances using the Dijet Mass Spectrum with 19.6 $\mathrm{fb}^{-1}$ of pp Collisions at $\sqrt{s}=8 \mathrm{TeV}$ ", note CMSPAS-EXO-12-059, Feb. 2013.

[26] C.W. Bauer, et al, "Supermodels for early LHC," Phys. Lett. B 690, 280 (2010) arXiv:0909.5213.

[27] A. V. Manohar and M. B. Wise, "Flavor changing neutral currents, an extended scalar sector, and the Higgs production rate at the LHC," Phys. Rev. D 74, 035009 (2006) hep-ph/0606172.

[28] K. S. Babu, C. F. Kolda and J. March-Russell, "Leptophobic $\mathrm{U}(1) s$ and the $\mathrm{R}(b)$ - $\mathrm{R}(c)$ crisis," Phys. Rev. D 54, 4635 (1996) [hep-ph/9603212.

[29] H. Georgi and S. L. Glashow, "Decays of a leptophobic gauge boson," Phys. Lett. B 387, 341 (1996) hep-ph/9607202.

[30] J. L. Rosner, "Prominent decay modes of a lep- tophobic $Z^{\prime}$," Phys. Lett. B 387, 113 (1996) hep-ph/9607207.

[31] P. Fileviez Perez and M. B. Wise, "Breaking local baryon and lepton number at the TeV scale," JHEP 1108, 068 (2011) arXiv:1106.0343.

[32] C. T. Hill, "Topcolor: Top quark condensation in a gauge extension of the standard model," Phys. Lett. B266, 419-424 (1991) ;

C. T. Hill, S. J. Parke, "Top production: Sensitivity to new physics," Phys. Rev. D49, 44544462 (1994). hep-ph/9312324.

[33] R. S. Chivukula, A. G. Cohen, E. H. Simmons, "New strong interactions at the Tevatron?," Phys. Lett. B380, 92-98 (1996). hep-ph/9603311;

E. H. Simmons, "Coloron phenomenology," Phys. Rev. D55, 1678-1683 (1997). hep-ph/9608269.

[34] Y. Bai and B. A. Dobrescu, "Heavy octets and Tevatron signals with three or four b jets," JHEP 1107, 100 (2011) arXiv:1012.5814.

[35] R. S. Chivukula, E. H. Simmons and N. Vignaroli, "A Flavorful Top-Coloron Model," Phys. Rev. D 87, 075002 (2013) arXiv:1302.1069; "Same-sign dileptons from colored scalars in the Flavorful Top-Coloron Model", arXiv:1306.2248,

[36] K. Kumar, R. Vega-Morales and F. Yu, "Effects from New Colored States and the Higgs Portal on Gluon Fusion and Higgs Decays," Phys. Rev. D 86, 113002 (2012) arXiv:1205.4244.

[37] P. Batra, B. A. Dobrescu and D. Spivak, "Anomaly-free sets of fermions," J. Math. Phys. 47, 082301 (2006) hep-ph/0510181.

[38] M. S. Carena, A. Daleo, B. A. Dobrescu and T. M. P. Tait, " $Z$ ' gauge bosons at the Tevatron," Phys. Rev. D 70, 093009 (2004) 
hep-ph/0408098. M. Duerr, P. Fileviez Perez and M. B. Wise, "Gauge Theory for Baryon and Lepton Numbers with Leptoquarks," Phys. Rev. Lett. 110, 231801 (2013) arXiv:1304.0576 [hep$\mathrm{ph}]]$.

[39] C. D. Carone and H. Murayama, "Possible light $\mathrm{U}(1)$ gauge boson coupled to baryon number," Phys. Rev. Lett. 74, 3122 (1995) hep-ph/9411256; "Realistic models with a light U(1) gauge boson coupled to baryon number," Phys. Rev. D 52, 484 (1995) hep-ph/9501220.

P. Fileviez Perez and M. B. Wise, "Baryon and lepton number as local gauge symmetries," Phys. Rev. D 82, 011901 (2010) [Erratum-ibid. D 82, 079901 (2010)] arXiv:1002.1754.

[40] S. Chatrchyan et al. [CMS Collaboration], "Searches for Higgs bosons in pp collisions at $\operatorname{sqrt}(\mathrm{s})=7$ and $8 \mathrm{TeV}$ in the context of fourgeneration and fermiophobic models," Phys. Lett. B 725, 36 (2013) arXiv:1302.1764 [hepex]]. ATLAS Collaboration, "Update of the Combination of Higgs Boson Searches in pp Collisions at $\sqrt{s}=7 \mathrm{TeV}$," note ATLAS-CONF2011-135.

[41] G. Aad et al. [ATLAS Collaboration], "Search for pair production of heavy top-like quarks decaying to a high- $p_{T} W$ boson and a $b$ quark in the lepton plus jets final state at $\sqrt{s}=7 \mathrm{TeV}$ ", Phys. Lett. B 718, 1284 (2013) arXiv:1210.5468;

S. Chatrchyan et al. [CMS Collaboration], "Search for heavy, top-like quark pair production in the dilepton final state in $p p$ collisions at $\sqrt{s}=7$ TeV," Phys. Lett. B 716, 103 (2012) arXiv:1203.5410; "Search for pair produced fourth-generation up-type quarks in $p p$ collisions at $\sqrt{s}=7 \mathrm{TeV}$ with a lepton in the final state,"
Phys. Lett. B 718, 307 (2012) arXiv:1209.0471.

[42] S. Chatrchyan et al. [CMS Collaboration], "Search for heavy bottom-like quarks in 4.9 inverse femtobarns of $p p$ collisions at $\sqrt{s}=7 \mathrm{TeV}$," JHEP 1205, 123 (2012) arXiv:1204.1088; "Search for heavy quarks decaying into a top quark and a $W$ or $Z$ boson using lepton + jets events in $p p$ collisions at $\sqrt{s}=7 \mathrm{TeV}$," JHEP 01, 154 (2013) arXiv:1210.7471]; "Combined search for the quarks of a sequential fourth generation," Phys. Rev. D 86, 112003 (2012) arXiv:1209.1062.

[43] L. J. Hall and A. E. Nelson, "Heavy Gluons And Monojets," Phys. Lett. B 153, 430 (1985); P. H. Frampton and S. L. Glashow, "Chiral Color: An Alternative to the Standard Model," Phys. Lett. B 190, 157 (1987).

[44] B. A. Dobrescu, K. Kong and R. Mahbubani, "Prospects for top-prime quark discovery at the Tevatron," JHEP 0906, 001 (2009) arXiv:0902.0792.

[45] B. A. Dobrescu, K. Kong and R. Mahbubani, "Massive color-octet bosons and pairs of resonances at hadron colliders," Phys. Lett. B 670, 119 (2008) arXiv:0709.2378.

[46] C. Quigg, "LHC Physics Potential versus Energy," arXiv:0908.3660 [hep-ph].

[47] S. Chatrchyan et al. [CMS Collaboration], "Data Parking and Data Scouting at the CMS Experiment," CMS-DP-2012-022.

[48] R. S. Chivukula, A. Farzinnia, E. H. Simmons and R. Foadi, "Production of massive color-octet vector bosons at Next-to-Leading Order," Phys. Rev. D 85, 054005 (2012) arXiv:1111.7261. R. S. Chivukula, A. Farzinnia, J. Ren and E. H. Simmons, "Hadron Collider Production of 
Massive Color-Octet Vector Bosons at Next-to-

Leading Order," Phys. Rev. D 87, 094011 (2013) arXiv:1303.1120 [hep-ph]].

[49] J. Alwall, et al, "MadGraph 5 : Going Beyond," JHEP 1106, 128 (2011) arXiv:1106.0522.

[50] J. Pumplin, et al, "New generation of parton distributions with uncertainties from global QCD analysis," JHEP 0207, 012 (2002) hep-ph/0201195.

[51] T. Sjostrand, S. Mrenna and P. Z. Skands, "PYTHIA 6.4 Physics and Manual," JHEP 0605, 026 (2006) hep-ph/0603175.

[52] J. Conway et al, "Pretty Good Simulation of high energy collisions", http://physics.ucdavis. edu/ conway/research/software/pgs /pgs4-general.htm

[53] G. Z. Krnjaic, "Very light axigluons and the top asymmetry," Phys. Rev. D 85, 014030 (2012) arXiv:1109.0648.

M. Gresham, J. Shelton and K. M. Zurek, "Open windows for a light axigluon explanation of the top forward-backward asymmetry," JHEP 1303, 008 (2013) arXiv:1212.1718.
[54] F. Yu, "A $Z^{\prime}$ model for the CDF dijet anomaly," Phys. Rev. D 83, 094028 (2011) arXiv:1104.0243.

[55] D. Choudhury, R. M. Godbole and P. Saha, "Dijet resonances, widths and all that," JHEP 1201, 155 (2012) arXiv:1111.1054 [hep-ph]].

[56] A. Atre, et al, "Probing color octet couplings at the LHC", Phys. Rev. D 86, 054003 (2012) arXiv:1206.1661.

[57] T. Aaltonen et al. [CDF Collaboration], "Search for the production of narrow $t \bar{b}$ resonances in 1.9 $\mathrm{fb}^{-1}$ of $p \bar{p}$ collisions at $\sqrt{s}=1.96 \mathrm{TeV}$," Phys. Rev. Lett. 103, 041801 (2009) arXiv:0902.3276.

[58] A. R. Zerwekh, "Axigluon Couplings in the Presence of Extra Color-Octet Spin-One Fields," Eur. Phys. J. C 65, 543 (2010) arXiv:0908.3116 [hep-ph]]. "Constraining Spin-One Color-Octet Resonances Using CDF and ATLAS Data," Eur. Phys. J. C 70, 917 (2010) arXiv:1008.4575 [hep$\mathrm{ph}]$.

[59] J. Gaiser, "Charmonium spectroscopy from radiative decays of the $J / \psi$ and $\psi^{\prime}$," SLAC-255 (1983), see Appendix F.1. 